\title{
Organoprotective Effect of Remote Ischemic Preconditioning during Living Donor Liver Transplantation, A Prospective Randomized Double-Blinded Study
}

\author{
Alrefaey K Alrefaey, Mohammed A Elmorshedi, Usama A Elsayed
}

Department of Anesthesia and Surgical Intensive Care, Faculty of Medicine, Mansoura University, Egypt

Address for Correspondence: Alrefaey K. Alrefaey,Email: refa3ey2@yahoo.com , Mobile:00201064203475

\begin{abstract}
Background: Ischemia reperfusion injury (IRI) during liver transplantation carries a substantial risk for graft damage, and other major organ injury. Strategies to minimize IRI in the grafted liver are of paramount importance. Remote ischemic preconditioning (RIP) is a recently described technique that can offer a liver protective effect against IRI.

Patients and Methods: In this prospective randomized study, consent was obtained from 80 couples (donorrecipient) prepared for liver transplantation (LT). Patients were divided randomly (using closed envelope technique) into two groups: RIP group ( $n=35)$ as RIP procedure was run in the donor non-dominant hand just at the start of parenchymal transection and control group $(n=35)$. In all the study cases, the pressure cuff was wrapped around the recipient arm. In RIP group, four sets of 5 minutes manual pressure cuff elevation to $200 \mathrm{mmHg}$, separated by 5 minutes of release (Using the non-dominant hand).

Results: No statistically significant difference was found between the biochemical profile (SGPT, CRP, and bilirubin) of the recipients in both of the study groups. Similarly, post-operative kidney function (Serum creatinine) was comparable findings in the two studied groups.

Conclusion: RIP in living donor liver transplantation failed to show a protective effect neither on the graft response to reperfusion injury, nor the on the postoperative kidney functions. Future studies are recommended taking into consideration histopathological, tissue damage scores.
\end{abstract}

Keywords: Liver transplantation, Preconditioning, Reperfusion, Organ protection.

\section{INTRODUCTION}

Ischemia-reperfusion injury (IRI) is a condition where the tissue and organ damage initiated during an ischemic episode is accentuated when blood flow and oxygen delivery is resumed. The pathophysiology of IRI involves direct ischemic cell injuries that trigger a cascade of inflammatory response ${ }^{(1)}$. The extent of damage extends beyond the primary affected organ to cause a subsequent remote organs dysfunction ${ }^{(2-5)}$. IRI was encountered in a large variety of clinical situations including stroke, myocardial ischemia, limb ischemia, kidney transplantation and liver transplantation. In the era of the shortage in liver donor and the use of marginal grafts, strategies to minimize IRI in the grafted liver the effect are of paramount importance ${ }^{(1)}$.

In ischemic preconditioning (IPC), the adaptation of the target organ tissues to prolonged ischemic insult and subsequent reperfusion injury is induced through short periods of planned ischemia. IPC has been used in many experimental models to protect the liver from IRI where results do not only support its ability to protect the liver against IRI but also enhances the capacity of liver regeneration ${ }^{(4)}$. Also, in liver surgery, this can be done either by local application of ischemia (pringle maneuver) or by remote organ ischemia (intestine, upper limb or lower $\operatorname{limb}{ }^{(6,7)}$.

In this study, the effect of remote IPC (RIP) of the donor upper limb on IRI of the liver graft was assessed in the setting of living donor liver transplantation. Early graft functions in the form of Liver enzymes (primary variable), serum bilirubin and C-reactive protein in the $1^{\text {st }}$ post-transplant week was considered as an indicator of graft IRI. In addition, the study investigated the effect of RIP on post-transplant kidney function, and the occurrence of acute kidney injury (AKI) in liver transplant recipients (secondary variables).

\section{PATIENTS AND METHODS}

In this prospective randomized study, consent was obtained from 80 couples (donor-recipient) prepared for liver transplantation (LT). Recruited recipients were of either sex, age ranged from 20 to 50 years, body mass index (BMI) was $\leq 35 \mathrm{~kg} / \mathrm{m}^{2}$, and model for end-stage liver disease (MELD) score was $\leq$ 25. Donors were of either sex, age ranged from 18-35 years and BMI was $\leq 30 \mathrm{~kg} / \mathrm{m}^{2}$.

Patients with known peripheral vascular disease, graft to patient-weight ratio (GRWR) was $\leq$ $0.7 \mathrm{gm} / 100 \mathrm{gm}$ (either by preoperative volumetry or direct intraoperative calculation), the need of high dose vasopressor infusion before graft reperfusion, postreperfusion vascular occlusion, and patients with postoperative pathological diagnosis of acute rejection were excluded from the study.

Patients were divided randomly (using closed envelope technique) into two groups: RIP group $(n=35)$ as RIP procedure was run in the donor non- 
dominant hand just at the start of parenchymal transection, and control group $(\mathrm{n}=35)$.

\section{Ethical approval}

The study was approved of Institutional Review Board of Mansoura Medicine School, Mansoura University, Egypt (R/15.08.63), and clinical trial registry (NCT02534025).

\section{Remote ischemic preconditioning procedure:}

To ensure blinding, both the patient and the investigator were blinded for the procedure. RIP was done by a different investigator who was not involved in patient' monitoring. In all the study cases, the pressure cuff was wrapped around the recipient arm. Four sets of 5 minutes manual pressure cuff elevation to $200 \mathrm{mmHg}$, separated by 5 minutes of release (Using the non-dominant hand). If RIP was not applied, the cuff tubing was clamped, and the investigator simulated doing the procedure without an actual application of the pressure.

\section{Anesthetic management:}

Routine preoperative anesthetic assessment was done according to the local protocol of Mansoura liver transplantation program including medical and anesthetic history, clinical examination, and laboratory investigations. On the day of surgery, the donors were premedicated with IV infusion of $40 \mathrm{mg}$ pantoprazole. The induction was done after the connection of basic monitors (ECG-SpO2-NIBP) using fentanyl $2 \mu \mathrm{g} / \mathrm{kg}$, rocuronium $0.6 \mathrm{mg} / \mathrm{kg}$ and Propofol $1-2 \mathrm{mg} / \mathrm{kg}$. After insertion and fixation of proper sized ETT, side-stream capnography was attached. The patient was ventilated using Aisys workstation (GE, Germany). Ultrasound guided internal jugular central line and radial artery catheter was inserted for monitoring and sampling. Concurrently two large bore cannulae were introduced to suitable peripheral veins. Anesthesia was maintained by sevoflurane inhalation, rocuronium and fentanyl infusion. Both rocuronium and fentanyl infusion were stopped one hour before skin closure, while sevoflurane was stopped with skin closure then extubation was done in OR faster fulfilling criteria of extubation unless there was a clinical need for ongoing respiratory support.

Upon recovery, all patients were transferred to the intensive care unit. Donors were admitted to ICU for 72 hours postoperatively. For the recipients, standardized immunosuppression regimen was commenced on day 1 post-operatively. Platelets and fresh frozen plasma were administered if there was a coagulopathy associated with active blood loss guided by ROTEM test ${ }^{(8)}$.

Thromboprophylaxis commenced on the first post-operative day unless contraindicated. All patients underwent a daily laboratory assessment of liver and

kidney functions plus a Doppler ultrasound scans of the graft and its vasculature anastomosis.

\section{Sample size and Statistical analysis:}

$\mathrm{G}^{*}$ power software version 3.1.9.2 was used for sample size calculation. Using the results from previously transplant cases in our center, remote ischemic preconditioning was assumed to decrease the postoperative SGPT level (mean $240 \mathrm{U} / \mathrm{dl}$ ) by $20 \%$. A total number of 56 cases were sufficient to get a power of $80 \%$. More 4 cases were recruited to compensate for dropouts, giving a total number of 60 cases, 30 per each group. Data were collected, tabulated and analyzed using SPSS software version 20. Continuous data were presented as mean \pm SD if normally distributed or median (IQR) if not. Nominal and ordinal data were presented as numbers/proportions. Independent sample T-test, Mann-Whitney test, or chisquared test was used for comparison between the study groups as appropriate.

\section{RESULTS}

In this study, eighty patients were recruited for enrollment in the study. After exclusion of patients not meeting the enrollment criteria, seventy patients completed the study protocol (figure 1). Patients basal and perioperative characteristics are listed in table (1).

The post-operative laboratory results and graft function are recorded in table (2), where no statistically significant difference was found between the biochemical profile (SGPT, C-RP and bilirubin) of the recipients in both the study groups. Similarly, postoperative kidney function (serum creatinine) was demonstrated in figure (2) and figure (3) showing comparable findings in the two studied groups.

Table (1): Patients basal and perioperative characteristics in the studied groups (Data are presented as mean $\pm \mathrm{SD}$ )

\begin{tabular}{lll}
\hline & $\begin{array}{c}\text { Control Group } \\
(\mathrm{n}=35)\end{array}$ & $\begin{array}{l}\text { RIP Group } \\
(\mathrm{n}=35)\end{array}$ \\
\hline Age (years) & $46 \pm 13.4$ & $49.6 \pm 12.4$ \\
Weight (Kg) & $80.9 \pm 16.3$ & $83.9 \pm 16.7$ \\
Height (m) & $1.67 \pm 0.11$ & $1.66 \pm 0.12$ \\
MELD score & $13.19 \pm 4.61$ & $15.33 \pm 2.9$ \\
Child-Pugh Score & $8.3 \pm 1.6$ & $8.6 \pm 1.8$ \\
Preoperative & $0.91 \pm 0.29$ & $0.87 \pm 0.29$ \\
Creatinine (mg/dl) & & \\
CI time (minutes) & $32 \pm 15.7$ & $29 \pm 14$ \\
WI time (minutes) & $38.2 \pm 8.74$ & $39.5 \pm 12.33$ \\
Operative time & $595.16 \pm 83.6$ & 613.28 \\
(minutes) & & 78.49 \\
Blood loss (liter) & $8.23 \pm 7.1$ & $6.95 \pm 6.23$ \\
\hline \multicolumn{2}{l}{ MELD: Model for End-Stage Liver } & Disease, Cold \\
ischemia, WI: warm ischemia &
\end{tabular}


https://ejhm.journals.ekb.eg/

Table (2): Postoperative biochemical profile and graft function. Data are presented as median (interquartile range), mean $\pm \mathrm{SD}$

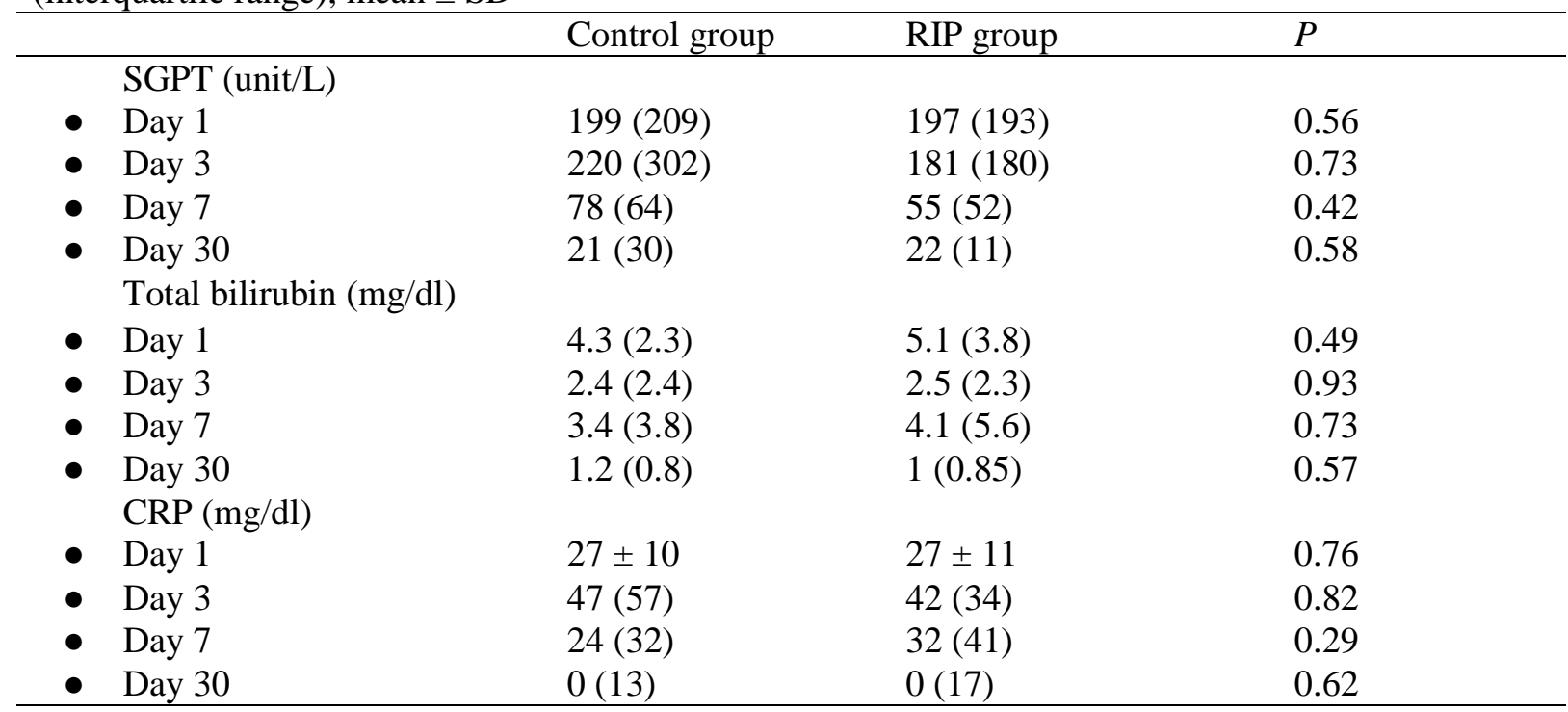

SGPT: serum glutamic-pyruvic transaminase , CRP: C reactive protein

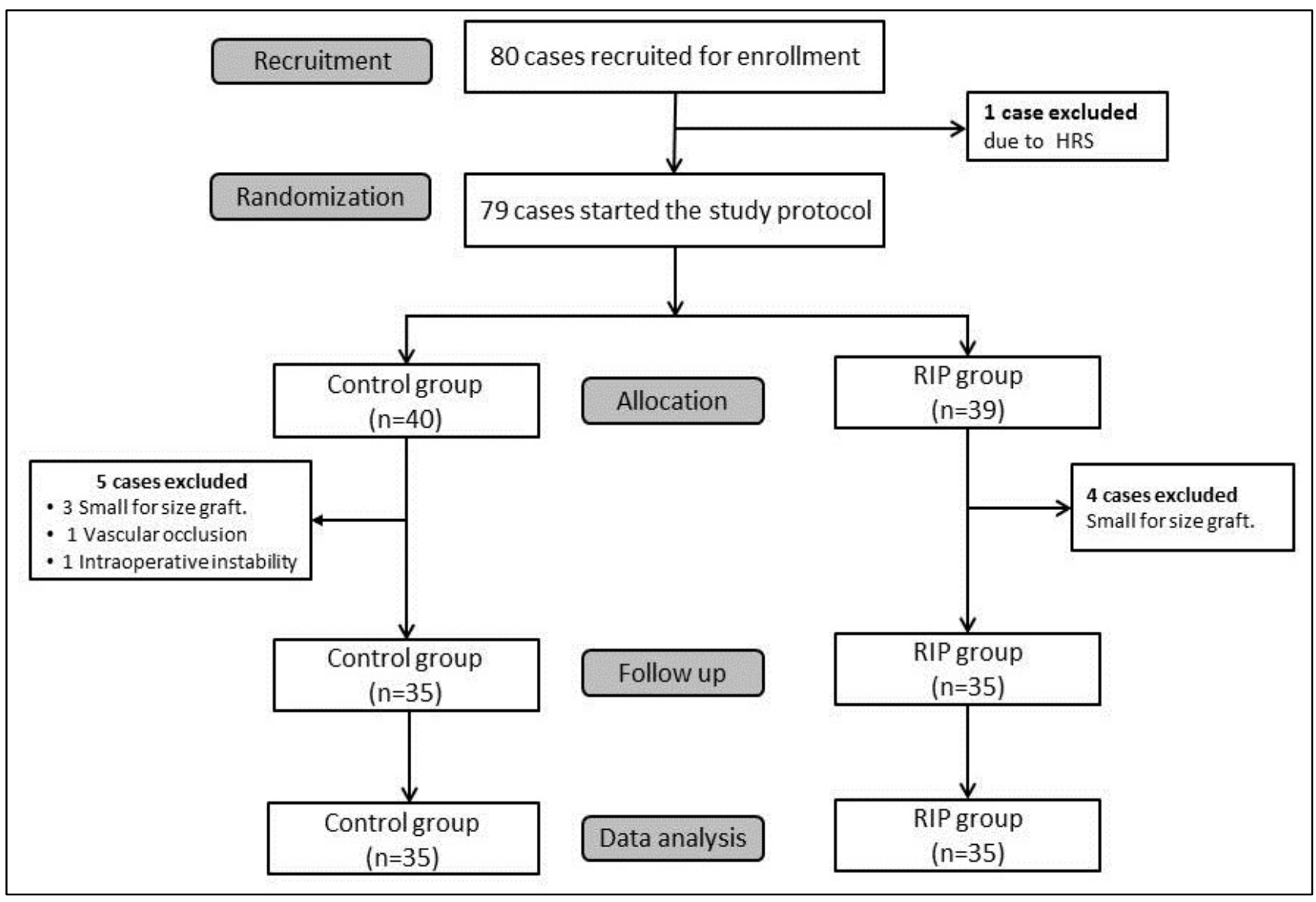

Figure (1): Study consort flow chart 


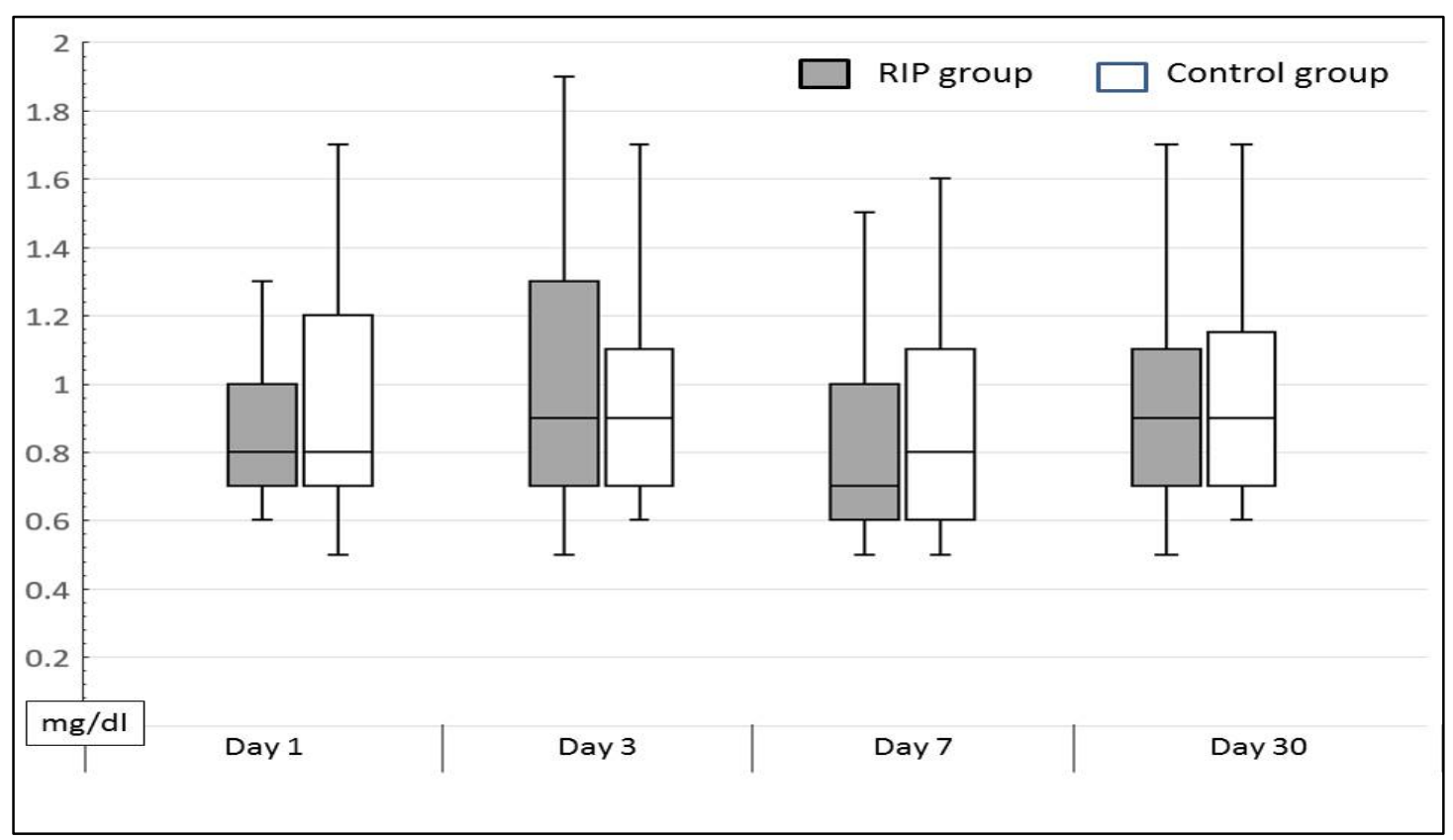

Figure (2): Boxplot for the postoperative kidney function in the study groups

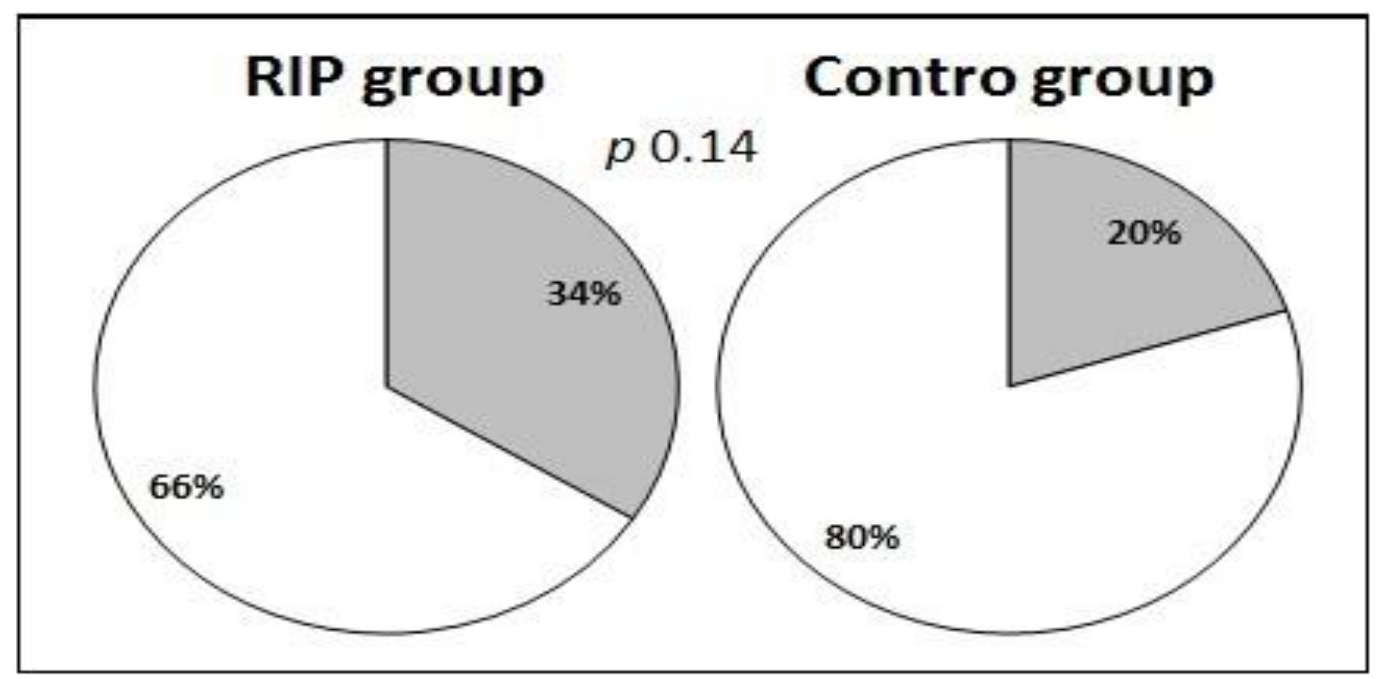

Figure (3): The early incidence AKI among the two study groups

\section{DISCUSSION}

In our study, seventy liver transplant cases were assessed for the organ protective effect of RIP before organ procurement through assessment of the graft reperfusion injury and postoperative renal function. Ischemia reperfusion injury of the grafted liver was assessed using the postoperative biochemical profile, where the RIP could not show a statistically significant protective effect. In addition, graft function, and postoperative incidence of renal impairment did not show statistically significant difference between the two studied groups.

In liver transplantation, the exposure of the grafted liver to IRI is a serious hazard affecting the grafted organ. After the hypoxic organ damage, restoration of blood flow and oxygen delivery results in the activation of inflammatory cascades with subsequently delayed dysfunction and tissue injury ${ }^{(9}$, ${ }^{10)}$. IRI had been linked to poor graft function in cases with prolonged ischemia after liver transplantation. After ischemic period, tissue damage may be aggravated during the reperfusion phase via the production of reactive oxygen radicals and the activation of phospholipase A2 by calcium influx during the hypoperfusion phase. IRI can extend beyond its local effect on the transplanted liver but also can extend to induce acute kidney injury (AKI) and serious cardiac complications ${ }^{(7,11)}$.

Ischemic preconditioning techniques were utilized to ameliorate the deleterious effect of IRI, 
especially in marginal graft sizes. One of the common techniques is the Pringle's maneuver, which represents a valuable method to decrease the blood loss and transfusion requirements in hepatic resection, thus facilitating the technical development of liver transplantation. However, direct liver preconditioning can impair the graft regeneration. The precise mechanisms by which IPC confers protection to the liver against IRI are still undetermined. However, studies postulated that the mechanism may be the preservation of ATP stores, activation of antioxidant systems, control of harmful mediator production like TNF- $\alpha$ synthesis and nitric oxide (NO) synthesis ${ }^{(4,5)}$.

Different strategies of ischemic preconditioning were explored in animal models and during liver transplantation surgeries. Despite the promising protective effects in animal models, results in human trials were debatable and sometimes claimed to have detrimental effect ${ }^{(12)}$. In an analysis of six different studies of IPC in liver transplantation, Desai et al. ${ }^{(13)}$ found that four of the studies reported a lower reperfusion injury, while one study reported no effect, and one of the studies reported a more IRI. In a randomized prospective study with 60 liver transplant patients, IPC for 10 minutes could decrease cellular apoptosis and significantly improve the reperfusion injury ${ }^{(14)}$. During their study of IPC in deceased donor liver transplantation, a 5 minutes clamping of the hilum in the donor failed to reduce graft injury ${ }^{(15)}$. Furthermore, longer ischemia time reaching $10 \mathrm{~min}$ resulted in higher ALT and AST levels in the early post-transplant period, which was considered as an IPC paradox as indicated by Koneru et al. ${ }^{(16)}$. Similar to the results of this study, five of the six studies did not show significant improvement in patient' outcome variables like length of stay, rejection, infections and graft' and patient' survival ${ }^{(14-19)}$. Likewise, even with a significantly lower ALT and AST in recipients receiving preconditioning, this biochemical enhancing effect did not affect early graft function ${ }^{(20)}$.

Acute kidney injury is not uncommon after liver transplantation ${ }^{(21)}$. The renoprotective effect of IPC was explored in animal and human studies with non-parallel results ${ }^{(20,22-24)}$. While, some of the studies supported the renoprotective effect of IPC ${ }^{(24)}$, others failed to demonstrate any beneficial effect ${ }^{(23)}$. In this study, early kidney function and the incidence of AKI was similar in the two study groups.

This study had some limitations. Liver biopsies and histopathological examination using specific scores like the Suzuki score ${ }^{(25)}$ could help to define the local effects of RIP on the liver graft. Additionally, serum levels of cytokines and other indicators of reperfusion injury could add to the precision of the study results.

The contradictory results of the studies investigating the effect of RIP may be due to the loss of the humoral mediators after the rinsing of the graft. In addition, graft conditioning near to the procurement process limits the time available for protective changes to settle and thus decrease its conditioning benefits. To boost the protective effect of RIP, some researchers recommended combined benefit of direct graft ischemic preconditioning and RIP while others advised conditioning for both the donor and the recipient ${ }^{(12,22)}$.

\section{CONCLUSION}

RIP in living donor liver transplantation failed to show any protective effect neither on the graft response to reperfusion injury, nor the protective effect on kidney functions. Future studies are recommended taking into consideration histopathological, tissue damage scores.

\section{REFERENCES}

1. Datta G, Fuller B, Davidson B (2013): Molecular mechanisms of liver ischemia reperfusion injury: Insights from transgenic knockout models. World Journal of Gastroenterology, 19: 1683-98

2. Elias M JC and Peralta C (2012): IschemiaReperfusion Injury Associated with Liver Transplantation in 2011: Past and Future, Liver Transplantation - Basic Issues, Prof. Hesham Abdeldayem (Ed.), Available from: http://www.intechopen.com

3. Costa F da S, Yamaki V, Gonçalves T et al. (2014): Combined remote ischemic perconditioning and local postconditioning on liver ischemia-reperfusion injury. The Journal of surgical research, 192: 98-102

4. Arumugam T, Magnus T, Woodruff T et al. (2006): Complement mediators in ischemia-reperfusion injury. Clinica Chimica Acta, (374): 33-45

5. Macedo F, Miranda $L$ (2010): Role of ischemic preconditioning in liver transplant: a review of literature. Experimental and Clinical Transplantation. Official Journal of the Middle East Society for Organ Transplantation, 8: 1-3

6. Tapuria N, Junnarkar S, Dutt $N$ et al. (2009): Effect of remote ischemic preconditioning on hepatic microcirculation and function in a rat model of hepatic ischemia reperfusion injury. HPB : the official journal of the International Hepato Pancreato Biliary Association, 11: 108-17

7. Yan S, Jin L, Liu Y et al. (2010): Outcomes and mechanisms of ischemic preconditioning in liver 2010transplantation. Hepatobiliary Pancreat Dis Int., 9: $346-54$

8. Kandeel A, Abdalla U, Abouelella M et al. (2018): Rotational thormboelastolmetry guided transfusion practice in living donor liver transplantation, A retrospective comparative study. Egyptian Journal of 
Anaesthesia, 34: 55-9

9. Nastos C, Kalimeris K, Papoutsidakis N et al. (2014): Global consequences of liver ischemia/reperfusion injury. Oxid Med Cell Longev., 2014:1-13

10. Montalvo-Jave E, Piña E, Montalvo-Arenas C et al. (2009): Role of ischemic preconditioning in liver surgery and hepatic transplantation. Journal of Gastrointestinal Surgery, 13: 2074-83

11. Aguilar-Melero $P$, Luque $A$, Machuca $M$ et al. (2013): Cardiotrophin-1 reduces ischemia/reperfusion injury during liver transplant. Journal of Surgical Research, 181: 83-91

12. Farooqui W, Pommergaard H, Rasmussen A (2018): Remote ischemic preconditioning of transplant recipients to reduce graft ischemia and reperfusion injuries: A systematic review. Transplantation Reviews, 32: 10-15

13. Desai K, Dikdan G, Shareef A et al. (2008): Ischemic preconditioning of the liver: A few perspectives from the bench to bedside translation. Liver Transplantation, 14: 1569-77

14. Amador A, Grande L, Martí J et al. (2007): Ischemic Pre-conditioning in Deceased Donor Liver Transplantation: A Prospective Randomized Clinical Trial. American Journal of Transplantation, 7: 2180-9

15. Koneru B, Fisher A, He Y et al. (2005): Ischemic preconditioning in deceased donor liver transplantation: A prospective randomized clinical trial of safety and efficacy. Liver Transplantation, 11: 196-202

16. Koneru B, Shareef A, Dikdan G et al. (2007): The Ischemic Preconditioning Paradox in Deceased Donor Liver Transplantation-Evidence from a Prospective Randomized Single Blind Clinical Trial. American Journal of Transplantation, 7: 2788-96

17. Cescon M, Grazi G, Grassi A et al. (2006): Effect of ischemic preconditioning in whole liver transplantation from deceased donors. A pilot study. Liver Transplantation, 12: 628-35

18. Jassem W, Fuggle S, Cerundolo L et al. (2006): Ischemic preconditioning of cadaver donor livers protects allografts following transplantation. Transplantation, 81: 169-74

19. Azoulay D, Del Gaudio M, Andreani P et al. (2005): Effects of 10 minutes of ischemic preconditioning of the cadaveric liver on the graft's preservation and function: the ying and the yang. Annals of surgery, 242: 133-9.

20. Jung K-W, Kang J, Kwon H-M et al. (2019): Effect of Remote Ischemic Preconditioning Conducted in Living Liver Donors on Postoperative Liver Function in Donors and Recipients Following Liver Transplantation. Annals of Surgery, 271: 646-653

21. Abdel-Khalek E, Alrefaey A, Yassen A et al. (2018): Renal Dysfunction after Living-Donor Liver Transplantation: Experience with 500 Cases. Journal of Transplantation, 2018:1-9

22. Li D, Liu W, Wang G et al. (2018): Impact of combined ischemic preconditioning and remote ischemic perconditioning on ischemia-reperfusion injury after liver transplantation. Scientific Reports, 8: 17979

23. Desai K, Mora-Esteves C, Holland B et al. (2014): Does liver ischemic preconditioning in brain death donors induce kidney preconditioning? a retrospective analysis. Transplantation, 97: 337-43

24. Kim W, Lee J, Ko J et al. (2014): Effect of remote ischemic postconditioning on patients undergoing living donor liver transplantation. Liver Transplantation, 20: 1383-92

25. Saeed W, Jun D, Jang $K$ et al. (2017): Does necroptosis have a crucial role in hepatic ischemiareperfusion injury? PLoS ONE, 12: 1-12. 